\title{
Lumbar Epidural Anesthesia in the Treatment of Discal Lombosciatalgia: A Comparative Clinical Study between Methylprednisolone and Methylprednisolone with Levobupivacaine
}

\author{
Fátima Aparecida Emm Faleiros Sousa ${ }^{1}$, Orlando Carlos Gomes Colhado ${ }^{2}$
}

\begin{abstract}
Summary: Sousa FAEF, Colhado OCG - Lumbar Epidural Anesthesia in the Treatment of Discal Lombosciatalgia: A Comparative Clinical Study between Methylprednisolone and Methylprednisolone with Levobupivacaine.

Background and objectives: Lumbar epidural technique has been used in the treatment of lombosciatalgia since 1953. In most cases, methylprednisolone is used along with a local anesthetic, and it is not known whether the isolated use of methylprednisolone is equally effective in relieving symptoms. The objective of this study was to compare the efficacy of two different solutions - methylprednisolone with saline and methylprednisolone with levobupivacaine injected in the epidural space to heal lombosciatalgia secondary to lumbar herniated disk.
\end{abstract}

Methods: Sixty individuals ASA I and II, of both genders, ages 18 to 65 years participated in this randomized, double-blind study over a period of one year. They underwent interlaminar lumbar epidural analgesia without radioscopic control to heal a lombosciatalgia and they were divided into two groups: G-M (methylprednisolone + saline) and G-M + L (methylprednisolone + levobupivacaine + saline) both at a volume of $10 \mathrm{~mL}$. Diagnosis was based on history, physical exam, and imaging exam (MRI). The Visual Analogue Scale (VAS) was applied in a total of two blockades, 15 days apart.

Results: A reduction in pain severity was observed in the methylprednisolone-levobupivacaine group, but without statistical significance.

Conclusions: The analgesic efficacy of the G-M + L solution was superior to that of the G-M solution in the treatment of discal lombosciatalgia regarding the shorter time to onset of analgesia, but this was not significant at the time of discharge, and both solutions were effective in the treatment of discal lombosciatalgia.

Keywords: Sciatica; Intervertebral Disk Displacement; Methylprednisolone; Anesthetics, Local; Analgesia, Epidural.

๑2011 Elsevier Editora Ltda. All rights reserved.

\section{INTRODUCTION}

In 1953, steroids were used for the first time in epidural administration when Lievre et al. ${ }^{1}$ administered steroids for relief of sciatic pain.

In the cohort of individuals who manifest chronic lumbar pain, 39\% have internal disk disruption, coinciding with the pain caused by discography, indicating the origin of discogenic pain. Acute peripheral tissue nociception leads to the intraspinal synthesis of prostaglandins by inducing ciclooxigenase-2

Received from Universidade de São Paulo (USP), Brazil.

1. Professor of Universidade de São Paulo (USP)

2. PhD in Sciences from USP; Professor of the Anesthesiology and Pain Discipline of the Medicine Department of Universidade Estadual de Maringá (UEM), Anesthesiologist and Responsible for the Pain Sector of Santa Casa de Maringá

Submitted on July 12, 2010.

Approved on February 28, 2011.

Correspondence to:

Dr. Orlando Carlos Gomes Colhado

Rua Marechal Deodoro 682/61

Zona 07

87030020 - Maringá, PR, Brazil

E-mail: esperanssoli@uol.com.br and the activation of phospholipase A2, resulting in hyperalgia. Corticosteroids are known to inhibit phospholipase-2 and the expression of ciclooxigenase-2 during inflammation. They also reduce the synthesis of prostaglandin ${ }^{2-4}$.

The elucidation of lumbar pain etiology is eminently clinical, and ancillary exams are indicated to prove the diagnostic hypothesis. Lumbar pain can be divided into three types: low lumbar pain, which involves every biomechanical pain between the last rib and the gluteal fold, such as nerve root irritation or exacerbation of chronic symptoms; lombosciatalgia, which irradiates from the lumbar region to one or both lower limbs; and sciatic pain with uni- or bilateral irradiation from the root of the thighs through the knees and, in most cases, reaching the ipsilateral foot. It may be associated or not with sensorial and/or motor deficit. In the tree cases it is possible to use the epidural technique with corticosteroids for pain relief ${ }^{5-7}$.

The structural cause of sciatic pain, such as herniated disk or foraminal stenosis may or may not be identified during the investigation, and imaging abnormalities can be seen in asymptomatic patients. Besides, it may not be correct to correlate lumbar pain with radiological findings even if they are in agreement with the distribution of the sciatic pain ${ }^{8}$.

Discogenic low back pain usually begins with a traumatic event, such as lifting weight or a sudden fall causing com- 
pression of one or more nerve roots. Epidural anesthesia with corticosteroids is performed by an anesthesiologist in $85 \%$ of cases. Methylprednisolone is the corticosteroid used more often, and the choice of local anesthetic varies. The benefits of this technique include pain relief, reduction in the consumption of analgesics, maintenance of work activities, and in some individuals, elimination of the need of surgery. Pain relief is greater in cases of short duration ( $<3$ months) ${ }^{9}$.

The objective of the present study was to compare the analgesic efficacy of two different solutions injected in the epidural space - methylprednisolone with saline and methylprednisolone with levobupivacaine - to heal lombosciatalgia secondary to discal radiculopathy.

\section{METHODS}

Initially, 69 patients with lower lumbar pain radiating to the corresponding lower limb (radicular due to herniated disk) were evaluated and diagnosed over a 1-year period. Diagnosis was based on their history, physical exam, and imaging exam (MRI). All subjects were informed about the study and if they agreed to participate they signed an informed consent. The study was approved by the Research Ethics Committee of Santa Casa de Maringá according to a report of the Medical Ethics Committee.

Inclusion criteria were as follows: individuals with lombosciatalgia with a diagnosis of herniated disk on MRI, who had already been treated with anti-inflammatories and/or opioids without improvement, with complaints of acute pain for more than three months. Exclusion criteria were: patients with tumors, infection and/or infectious lesion at the site of puncture, severe cardiopathy, diabetes, smokers, alcoholics, use of anticoagulants, gravidas with structural changes in the lumbar spine that interfered with the epidural technique, motor deficit or sphincter changes with surgical indication; and those patients without the necessary cognition to understand the protocol.

Patients were admitted to Santa Casa de Maringá after an 8 hour fasting period, being monitored with non-invasive blood pressure, pulse oximetry, and heart rate. At this time the Visual Analogue Scale (VAS) was applied to measure pain before the anesthesia.

A solution was prepared in a sterile field with a $5 \mathrm{~mL}$ syringe used to aspirate $2 \%$ lidocaine for infiltration of skin and deeper tissues, such as lumbar subcutaneous and paraligament tissues. A solution was then prepared with corticosteroid methylprednisolone $80 \mathrm{mg}+8 \mathrm{~mL}$ of saline solution $0.9 \%$ in the first group. In the second group we used $80 \mathrm{mg}$ methylprednisolone solution $+5 \mathrm{~mL}$ of bupivacaine $0.5 \%$ without epinephri$\mathrm{ne}+3 \mathrm{~mL}$ of saline $0.9 \%$, both in a $10 \mathrm{~mL}$ syringe

Patients were placed in the sitting position: antisepsis of the lumbar region with topic povidine was performed, the L4-L5 space was identified, the skin and paraligament were anesthetized followed by introduction of a number 10 spinocan needle until it reached the epidural space, which was identified by the loss of resistance technique with a $10 \mathrm{~mL}$ glass syrin- ge, and the solution was injected. The epidural technique was performed without radioscopic control.

After the blockade patients were transferred to the postanesthetic recovery room for observation. Patients in both groups were transferred to a room after being discharged from the post-anesthetic recovery room. If analgesic rescue was necessary, $100 \mathrm{mg}$ of tramadol IV every 6 hours was used. The Visual Analogue Scale was used before the blockade and 30 minutes afterwards in the recovery room; and in the hospital ward within the periods of 6,12 and 24 hours.

Occasional adverse events such as hypotension, nausea and vomiting were evaluated whenever patients were visited for data collection, being treated with hydration and vasopressor, ephedrine sulfate, oxygen catheter, and ondansetron, respectively. Expected but rare complications such as epidural hematoma or abscess were evaluated through neurologic assessment, such as unexpected sensorial or motor deficit or systemic signs such as fever.

Patients had to score in VAS on a $10 \mathrm{~cm}$ line in which the extremes corresponded to "no pain" and "worst pain possible" for the intensity level of their pain. Patients were instructed to mark the maximal pain intensity at $10 \mathrm{~cm}$ and the lower at $0 \mathrm{~cm}$. The other alternatives, which in the scale corresponded to the numbers 1 to 9 , were used to describe intermediate pain levels.

Anthropometric data were analyzed with the $\mathrm{R} 2.12$ software, and they were compared by the Mann-Whitney test for age, Student $t$ test for weight and height, and Fisher exact test for gender. Normalcy of data was determined by Shapiro-Wilk test.

The sample size was calculated for a $90 \%$ confidence and $10 \%$ margin of error.

VAS was applied to define the best therapeutic response in both groups. For clinical analysis the linear model of mixed effects (random and fixed effects) was used in the statistical analysis of data when the answers of an individual are grouped and assuming the independence between observations in the same group is not adequate to the level of significance of $5 \%$. The model was adjusted by the PROC MIXED of the SAS $^{\circledR} 9.0$ software.

No more than two blockades in a 15 day interval were performed; upon return of the patient new evaluations were performed, and a second blockade was not indicated in those who did not have any improvement of symptoms after the first blockade, being referred for a neurosurgeon. On the other hand, those who experienced pain improvement underwent a second blockade. Adjuvant techniques, such as acupuncture or physiotherapy were not used. Patients were oriented to return to the physician's office or Santa Casa de Maringá in case of worsening of their pain, and that they should not take any analgesic without the knowledge of the authors even in the first week after the second blockade.

\section{RESULTS}

Of the 69 patients evaluated and selected for the study, three were referred immediately after the first blockade to decom- 
pressive surgery, two in the G-M group and one in the $\mathrm{G}-\mathrm{M}+\mathrm{L}$ group since they did not show any improvement of their symptoms; and six patients gave up after the first blockade - three said they were doing fine and they did not need a second blockade, and three gave other reasons. Sixty of these patients, ASA I and II, of both genders, ages 18 to 65 years, were selected to participate in this randomized double-blind study. Mean age was 46.8 years. The level of greater incidence of a herniated disk was L4-L5. Regarding gender, females were more commonly affected, with 36 individuals out of 60 . The left limb was more commonly affected, with 35 individuals according to Table I. Since individuals were randomly selected a balanced result regarding age and weight was not obtained; as for height a significant difference was not observed. Table II shows the demographic data of the study population. Table III shows the results of VAS in the Methylprednisolone group (G-M) and Methylprednisolone + Levobupivacaine group $(G-M+L)$. In the $\mathrm{G}-\mathrm{M}+\mathrm{L}$ group after the first blockade pain severity differed significantly between "before" and the remaining times. Between the times "30 minutes and 24 hours", as well as " 6 hours and 24 hours" a significant difference was observed. After the second blockade the difference between "before" and the remaining times and between "30 minutes and 12 hours"

Table I - Drugs used, Level of Herniated Disk, and Lower Limb with Irradiation of the Lumbar Pain Regarding Gender

\begin{tabular}{llll}
\hline & Male & Female & Both genders \\
\hline Drug & & & \\
G-M + L & $17(70.83)$ & $13(36.11)$ & $30(50.00)$ \\
G-M & $7(29.17)$ & $223(63.89)$ & $30(50.00)$ \\
Level of herniated disk & & & \\
L3-L4 & $1(4.17)$ & $1(2.78)$ & $2(3.33)$ \\
L3-L4 L4-L5 & - & $3(8.33)$ & $3(5)$ \\
L4-L5 & $13(54.17)$ & $13(36.11)$ & $26(43.33)$ \\
L4-L5 L5-S1 & $1(4.17)$ & $5(13.89)$ & $6(10)$ \\
L5-S1 & $9(37.5)$ & $14(38.89)$ & $23(38.33)$ \\
Pain irradiation & & & \\
Lower right limb & $6(25)$ & $19(52.78)$ & $25(41.67)$ \\
Lower left limb & $18(75)$ & $17(47.22)$ & $35(58.33)$ \\
Total & $24(100)$ & $36(100)$ & $60(100)$ \\
\hline
\end{tabular}

Results expressed as percentages, G-M+L; methylprednisolone + levobupivacaine + saline $0.9 \%$, G-M; methylprednisolone + saline $0.9 \%$.

Table II - Demographic Data

\begin{tabular}{|c|c|c|c|}
\hline & $G-M+L$ & G-M & \\
\hline Age (years) * & $43.19 \pm 11.18$ & $49.00 \pm 10.32$ & 0.0356 \\
\hline Weight $(\mathrm{kg})$ * & $79.48 \pm 13.08$ & $75.66 \pm 13.18$ & NS \\
\hline Height $(m)$ * & $1.70 \pm 0.08$ & $1.65 \pm 0.07$ & 0.0038 \\
\hline Gender & & & 0.0084 \\
\hline Male & 17 & 7 & \\
\hline Female & 13 & 23 & \\
\hline
\end{tabular}

*Results expressed as Mean \pm Standard Deviation; G-M+L: methylprednisolone + levobupivacaine + saline $0.9 \%$; G-M: methylprednisolone + saline $0.9 \%$; NS: non-significant; Mann-Whitney test; Student $t$ test; Fisher exact test.
Table III - Descriptive Measurements of the Visual Analogue Scale Results per Block, Drug, and Moment

\begin{tabular}{|c|c|c|c|c|c|}
\hline & Mean & $\begin{array}{l}\text { Standard } \\
\text { Deviation }\end{array}$ & Minimum & Median & Maximum \\
\hline \multicolumn{6}{|l|}{ 1st block } \\
\hline \multicolumn{6}{|c|}{ G-M + L group } \\
\hline before ${ }^{a}$ & 6.22 & 2.51 & 0 & 7.25 & 10 \\
\hline $30 \min ^{b}$ & 3.89 & 2.91 & 0 & 3 & 10 \\
\hline $6 \mathrm{~h}^{\mathrm{b}}$ & 3.72 & 2.59 & 0 & 4 & 10 \\
\hline $12 h^{b c}$ & 3.39 & 2.43 & 0 & 3.5 & 8 \\
\hline $24 h^{c}$ & 2.79 & 2.11 & 0 & 2.75 & 7 \\
\hline \multicolumn{6}{|c|}{ G-M group } \\
\hline before ${ }^{a}$ & 5.68 & 2.69 & 0 & 5.75 & 10 \\
\hline $30 \min ^{b}$ & 4.34 & 2.62 & 0 & 4.5 & 9 \\
\hline $6 \mathrm{~h}^{\mathrm{bc}}$ & 3.85 & 2.91 & 0 & 3.5 & 9 \\
\hline $12 h^{c}$ & 3.33 & 2.5 & 0 & 3 & 8 \\
\hline $24 h^{c}$ & 3.36 & 2.53 & 0 & 2.75 & 9 \\
\hline \multicolumn{6}{|l|}{ 2nd block } \\
\hline \multicolumn{6}{|c|}{ G-M + L group } \\
\hline before $^{a}$ & 3.79 & 2.17 & 0 & 3.75 & 8.5 \\
\hline $30 \mathrm{~min}^{\mathrm{c}}$ & 1.86 & 1.95 & 0 & 1.5 & 7.5 \\
\hline $6 \mathrm{~h}^{\mathrm{bc}}$ & 2.19 & 2.16 & 0 & 1.7 & 7.5 \\
\hline $12 \mathrm{~h}^{\mathrm{b}}$ & 2.73 & 2.49 & 0 & 2 & 8.5 \\
\hline $24 h^{b c}$ & 2.13 & 2.03 & 0 & 1.5 & 7.5 \\
\hline \multicolumn{6}{|c|}{ G-M group } \\
\hline before ${ }^{a}$ & 3.65 & 2.44 & 0 & 3 & 10 \\
\hline $30 \min ^{b}$ & 2.75 & 2.72 & 0 & 2.2 & 10 \\
\hline $6 \mathrm{~h}^{\mathrm{b}}$ & 2.71 & 2.62 & 0 & 2 & 8 \\
\hline $12 h^{a b}$ & 2.94 & 2.99 & 0 & 2 & 8 \\
\hline $24 \mathrm{~h}^{\mathrm{b}}$ & 2.38 & 2.63 & 0 & 1.25 & 8.5 \\
\hline
\end{tabular}

G-M+L: methylprednisolone + levobupivacaine + saline 0.9\%, G-M: methylprednisolone + saline $0.9 \%$

*Different letters mean significant difference at the $5 \%$ level when compared within each group (block and drug), based on comparison of means in the model of mixed effects.

was also significant. In G-M, in the first blockade, "before" showed a significant difference from the remaining times as well as "30 minutes and 12 and 24 hours", and in the second blockade a significant difference was observed only between "12 hours" and the remaining times at the $5 \%$ level of significance.

The superscript letters (a, b, c, ab, and ${ }^{b c}$ ) give us the idea of a significant or lack of significant difference regarding the mean VAS. Whenever a moment received simultaneously bc or ab, it means a lack of significant difference between times ${ }^{b}$ and $/$ or $^{c}$, and ${ }^{a}$ or ${ }^{b}$. However, the times that received only ${ }^{b}$ differ from times ${ }^{a}$ and ${ }^{c}$ and vice-versa.

When comparing each time in both groups, a significant difference was not observed in the $5 \%$ level of significance and for this reason, no letter was used for comparison.

\section{DISCUSSION}

The present study demonstrated that both techniques - methylprednisolone alone and methylprednisolone associated with levobupivacaine - are effective in relieving discal compression pain when administered in the epidural space, but 
this was evaluated during a short-term, and both solutions can be used for this purpose. Addition of the local anesthetic levobupivacaine to methylprednisolone can reduce the latency of analgesia, which can be observed 30 minutes after the epidural injection. But this addition is not associated with better statistical results in pain relief when compared to methylprednisolone alone.

The role of epidural corticosteroids in the treatment of sciatic pain has been the center of discussions in the last 50 years, with studies reporting conflicting results. The therapeutic effect of epidural corticosteroids is attributed to the inhibition of synthesis or release of proinflammatory substances. Recent reviews on the epidural administration of corticosteroids report conflicting results, and a meta-analysis of 11 placebocontrolled studies showed a $75 \%$ improvement in pain both short-term (1-60 days) and long-term (12 weeks-1 year) concluding that this technique is effective in the treatment of low lumbar pain secondary to herniated lumbar disk ${ }^{10,11}$.

Hayashi et al. ${ }^{12}$ investigated the epidural technique with betamethasone and/or bupivacaine associated with saline in an animal model with nerve root irritation in 51 rats. Their results demonstrated that rats treated with betamethasone alone or associated with bupivacaine had significantly lower thermal hyperalgia $(p<0.01)$ than the group treated with saline or bupivacaine alone.

A study comparing the epidural transforamen administration of corticosteroids with saline injection in trigger points used in the treatment of lumbosacral radiculopathy due to herniated nucleus pulposus showed that after 1.4 year the group that received the epidural corticosteroid injection was successful in $84 \%$ of the individuals when compared to $48 \%$ in the saline group $(p<0.005)^{13}$.

After the epidural administration, local anesthetics cross the meninges to reach its site of action, which seems to be the dorsal and ventral spinal roots where they are leaving the spinal cord, being associated with sensorial and motor blockade related to its concentration. Future studies comparing the action of epidural corticosteroids with the systemic action of corticosteroids are necessary and urgent and if their action justifies only their systemic use it would not be worthwhile to continue using it in the interlaminar or transforamen route to control sciatic pain. Treatment of lombosciatalgia ranges from the conservative approach to surgery, and the inflammatory etiology justifies the use of epidural corticosteroids in its treatment.

In a retrospective study to assess the efficacy of the interlaminar injection of methylprednisolone and bupivacaine in the treatment of 60 individuals with lombosciatalgia who had no pain relief with rest and adequate drug treatment for at least one month, the authors concluded that interlaminar epidural injection of corticosteroids associated with bupivacaine can be beneficial for at least 6 months as an adjuvant technique in the conservative treatment of lombosciatalgia $10,14,15$.

All individuals initially selected to participate in the present study had lombosciatalgia related to a herniated disk, which was constricting the corresponding nerve root, and they all were evaluated by history, physical exam, and imaging exam (MRI) to confirm the diagnosis.

According to the American College of Physicians and the American Pain Society a careful history and physical exam is necessary to classify individuals with lumbar pain in one of three categories: non-specific lumbar pain, radiculopathy or spinal stenosis-associated lumbar pain, and lumbar pain possibly associated with another specific spinal cause. In the history, psychosocial risk factors, besides an individual evaluation of patients with persistent lumbar pain with signs and symptoms of radiculopathy or spinal stenosis, preferentially with MRI or CT, should be included ${ }^{16}$.

A study with 21 candidates for discectomy with sciatic pain with unilateral symptoms for two to four months to evaluate whether the epidural injection of corticosteroids could reduce the need for surgery demonstrated that the injection of betamethasone and lidocaine close to the nerve lesion was effective. Only three participants underwent surgical decompression for recurrent pain proving that this technique is simple, effective, and low risk, being an alternative to surgical decompression of herniated lumbar disk in selected cases ${ }^{17}$.

In the analysis of a randomized prospective multicenter study to determine whether individuals with severe sciatic pain for a short period would benefit from early surgery (during the first year) compared with prolonged conservative treatment with analgesics, the authors concluded that the group that underwent late surgery did not show differences in outcome when compared to those who underwent conservative treatment ${ }^{18}$.

In the present study major adverse effects such as nausea, vomiting, or cardiovascular or neurologic effects as well as inadvertent intravascular or subarachnoid injection were not observed in the 30 patients who received corticosteroids alone or associated with local anesthetic, since the dose of 25 $\mathrm{mg}$ of $0.25 \%$ levobupivacaine is low, and participants included in the study were ASA I or II.

Levobupivacaine and Ropivacaine, amino amide local anesthetics structurally similar to bupivacaine, were recently introduced. Both drugs have been associated with lower central nervous and cardiac toxicities than bupivacaine. Controlled, randomized studies comparing the tree drugs support the finding that the profile of levobupivacaine and ropivacaine is similar to that of racemic bupivacaine, and that the difference observed between the three anesthetics is related mainly to a slight difference in anesthetic potency, with bupivacaine $>$ levobupivacaine > ropivacaine. Levobupivacaine has two enantiomers: levobupivacaine $-S(-)$ and dextrobupivacaine $-\mathrm{R}(+)$.

Based on studies demonstrating that cardiotoxicity is lower with the $S(-)$ enantiomer the use of this agent in clinical practice is widespread being increasingly used in the epidural space for abdominal and lower limb surgeries due to its good local anesthetic action comparable to the racemic form and its low toxicity ${ }^{19-22}$.

In the present study the loss of resistance was confirmed with a glass syringe with $10 \mathrm{~mL}$ of air in the performance of the epidural technique. The volume of the solution injected 
in both groups, $0.25 \%$ levobupivacaine combined with $80 \mathrm{mg}$ of methylprednisolone and $0.9 \%$ saline in a volume of $8 \mathrm{~mL}$ associated with $80 \mathrm{mg}$ of methylprednisolone, was $10 \mathrm{~mL}$. L4-L5 space was chosen for the injection, since the greater incidence of herniated disk in this study was at the level of the L4-L5 and L5-S1 spaces (96.66\%), and two blockades were performed at a 15 day interval. The option to surgery was the epidural administration of corticosteroids, which is less invasive and has lower morbidity. Individuals were hospitalized for 24 hours and during this period they underwent six evaluations with VAS. We concluded that the group of methylprednisolone plus levobupivacaine had faster pain regression, being observed 30 minutes after the epidural injection, while in the methylprednisolone group more important pain relief was observed between 6 and 12 hours after the epidural injection; therefore, we concluded that this is the latency to action of this drug in this epidural space.

The therapeutic effect of epidural corticosteroids is attributed to the inhibition of the synthesis or release of proinflammatory substances, and a variety of corticosteroids (hydrocortisone, methylprednisolone, triamcinolone, and betamethasone) has been used in the neuraxis to treat pain of the same origin . In a randomized study with two groups of individuals with lumbar pain of radicular origin, $80 \mathrm{mg}$ of methylprednisolone in 43 patients was compared with $40 \mathrm{mg}$ of methylprednisolone in 41 individuals. They all received high doses of opioids, NSAIDS, and physiotherapy for more than 2 weeks before the epidural block. The drugs were administered in the L4-L5 space by the loss of resistance technique with a glass syringe with air. Visible improvement was observed in one month in 64 cases $(75 \%)$ by VAS. Both groups were compared at two weeks and three months after the epidural injection. Better results, although small, were observed in the group that received $40 \mathrm{mg}$ of methylprednisolone when compared to the $80 \mathrm{mg}$ group $(75.6 \%$ versus $74.4 \%)$ (,23.

Positive results for epidural corticosteroids range from $20 \%$ to $95 \%$ and depend on the route of administration, and the ones used more often include caudal, interlaminar, and transforamen. A study with 90 patients, 18 to 60 years old, with L5-S1 herniated disk documented by MRI and electroneuromyography, showed involvement of the S1 root.
Blockades were performed every two weeks for a maximal of three blockades, and patients were randomized by a computer. Triamcinolone $40 \mathrm{mg}(1 \mathrm{~mL})$ with $4 \mathrm{~mL}$ of saline was used in the interlaminar and transforamen technique, and $19 \mathrm{~mL}$ was used in the caudal technique, with all patients in the prone position during the blockade with a radioscopic device and use of contrast.

The visual analogue scale was used for the evaluation, and patients were reassessed two weeks after the initial blockade. Patients with complete or no pain relief did not undergo a new blockade.

The transforamen technique showed better results in pain relief followed by the interlaminar and caudal techniques. This result was attributed to the greater deposition of corticosteroid in the ventral portion of the epidural space ${ }^{24}$.

The interlaminar technique used in the present study was chosen due to the larger number of cases in literature with good results and the simplicity of the technique, since it forgoes the use of an image amplifier, with no exposure to radiation of both medical team and the patient, use of contrast, and radiotransparent surgical table.

We concluded that, although one of the groups had received the local anesthetic levobupivacaine in the epidural solution and this was responsible for the greater speed of the onset of analgesia this did not translate into more significant pain relief at the time of hospital discharge according to the statistical analysis, and since both groups received methylprednisolone this might explain the analgesic action of both solutions. We also concluded that the interlaminar epidural analgesic block is effective in the treatment of herniated diskinduced lombosciatalgia.

\section{ACKNOWLEDGEMENT}

The authors would like to acknowledge the collaboration of the anesthesiologists Marcelo Boeing, Luciano Bornia Ortega, and Patricia Reis, during the data collection phase, and to the statistician Ricardo Nonaka for his collaboration with the statistical analysis. 